\title{
Clinical impact of change of FLT3 mutation status in acute myeloid leukemia patients
}

\author{
Mikako Warren ${ }^{1}$, Rajyalakshmi Luthra ${ }^{2}$, C Cameron Yin ${ }^{2}$, Farhad Ravandi ${ }^{3}$, Jorge E Cortes ${ }^{3}$, \\ Hagop M Kantarjian ${ }^{3}$, L Jeffrey Medeiros $^{2}$ and Zhuang Zuo ${ }^{2}$ \\ ${ }^{1}$ Department of Pathology and Immunology, Baylor Collage of Medicine, Texas Children's Hospital, Houston, \\ TX, USA; ${ }^{2}$ Department of Hematopathology, The University of Texas M.D. Anderson Cancer Center, \\ Houston, TX, USA and ${ }^{3}$ Department of Leukemia, The University of Texas M.D. Anderson Cancer Center, \\ Houston, TX, USA
}

\begin{abstract}
FMS-like tyrosine kinase 3 (FLT3) is one of the most frequently mutated genes in acute myeloid leukemia and is associated with worse clinical outcome. Changes in FLT3 mutation status can occur during the course of disease, but the clinical impact of a change is unclear. We retrospectively reviewed 3555 acute myeloid leukemia patients, who have been assessed for FLT3 mutation at our institution between May 2002 and January 2011. We found that $42(6.2 \%)$ out of 680 patients with FLT3 mutation experienced a change of FLT3 mutation status. In all, 36 patients with wild-type $F L T 3$ at the time of initial diagnosis gained mutation (Negative/Positive) and six initially FLT3-mutated patients became wild type during their following relapses (Positive/Negative). The 5 -year survival of these patients was similar to that of patients with persistently wild-type FLT3 (Negative/ Negative; $P=0.464$ ), and significantly better than patients who had stable $F L T 3$ mutation during their disease course (Positive/Positive; $P<0.001$ ). However, after mutations became detectable in the Negative/Positive group, the forward survival of these patients tracked that of the Positive/Positive group after relapse $(P=0.761)$. In addition, we did not find a significant difference in survival between patients with internal tandem duplications and those with point mutations in the tyrosine kinase domain of the FLT3 gene. These results suggest that FLT3 mutations are unstable and that there is potential clinical value in continuously monitoring FLT3 mutation status.
\end{abstract}

Modern Pathology (2012) 25, 1405-1412; doi:10.1038/modpathol.2012.88; published online 8 June 2012

Keywords: acute myeloid leukemia; FMS-like tyrosine kinase (FLT3); mutation

The FMS-like tyrosine kinase 3 (FLT3) gene is located on chromosome $13 \mathrm{q} 12$ and encodes a membrane-bound receptor tyrosine kinase that has an important role in hematopoiesis. ${ }^{1-3}$ FLT3 is one of the most frequently mutated genes in hematological malignancies, present in over $30 \%$ of adults with acute myeloid leukemia. ${ }^{4-8}$ The most common type of FLT3 mutation is internal tandem duplications (FLT3-ITDs) in the juxtamembrane domain of the receptor, which have been found in $15-35 \%$ of adult acute myeloid leukemia patients. ${ }^{4,5,9,10}$ Point mutations in the heavily converted areas of the intracel-

Correspondence: Dr Zhuang Zuo, MD, PhD, The University of Texas M.D. Anderson Cancer Center, 8515 Fannin Street, NAO1.046a, Houston, TX 77054, USA.

E-mail: zzuo@mdanderson.org

Received 5 December 2011; revised 27 January 2012; accepted 27 January 2012; published online 8 June 2012 lular tyrosine kinase domain (TKD), most commonly the nucleotide substitution of aspirate 835 (FLT3D835), occur in $5-10 \%$ of adult acute myeloid leukemia patients. ${ }^{3,7,8,11}$ Although most patients have only one type of the FLT3 mutation, $1-3 \%$ of acute myeloid leukemia patients have both FLT3ITD and FLT-D835. ${ }^{8,11,12}$ FLT3-ITD and FLT-D835 cause constitutive activation of FLT3, leading to aberrant activation of multiple downstream pathways, such as phosphatidyl-inositol 3-kinase, mitogen-activated protein kinase, and signal transducer and activator of transcription $5 .{ }^{13}$

Patients with acute myeloid leukemia associated with FLT3 mutation usually present as de novo disease with high peripheral leukocyte count, high bone marrow blast count, and normal cytogenetics. ${ }^{14}$ FLT3-ITD is an independent predictor of poor prognosis and is associated with increased relapse risk after chemotherapy, and decreased disease-free 
survival and overall survival. ${ }^{6,8,10,15,16}$ The clinical significance of FLT3-D835 is still unclear. Some studies have shown that FLT3-D835 is associated with shorter disease-free and overall survival. ${ }^{7,14,17,18}$ Other studies did not identify differences in these parameters. ${ }^{8,14}$ Conflicting data may be due to small patient numbers, different treatment regimens, and patient selection.

The high frequency of FLT3 mutations in acute myeloid leukemia has made the FLT3 gene a promising target for developing therapeutic agents. There are several classes of FLT3 inhibitors currently in development or in clinical trials with varying degrees of potency and selectivity for the target. ${ }^{19}$ FLT3 inhibitors offer a potential paradigm shift in the standard therapy of acute myeloid leukemia patients. Some studies have even reported potential benefits of administering FLT3 inhibitors to acute myeloid leukemia patients without detectable FLT3 mutations. $^{20}$ One potential explanation is that FLT3 mutations are unstable, and that patients may acquire or lose mutations along the disease course.

Change of FLT3 status (gain or loss) at time of initial diagnosis and subsequently, usually at time of relapse, has been observed repeatedly in a small fraction of the patients in earlier studies. ${ }^{10,16,21-29}$ However, owing to a lack of a sufficient number of patients, no study has been able to provide statistical significant correlations between FLT3 status changes and disease progression. The goal of this study is to examine the clinical features and outcome of a large cohort of acute myeloid leukemia patients in whom FLT3 mutation status changed. In a retrospective review of 3555 acute myeloid leukemia patients, we identified 42 (6.2\%) with a change in FLT3 status. Our findings suggest that acquiring a FLT3 mutation during the course of disease has a negative impact on patient survival and may justify changes in clinical management.

\section{Materials and methods}

\section{Case Selection}

We retrospectively reviewed all clinical and laboratory data of all adult acute myeloid leukemia patients assessed for FLT3 mutation at the University of Texas M. D. Anderson Cancer Center between May 2002 and January 2011. All cases were diagnosed and classified as acute myeloid leukemia according to World Health Organization criteria. In all patients, FLT3 mutation testing was performed on bone marrow aspirate specimens obtained at initial diagnosis and at time of multiple follow-up visits. To assess the impact of a change in FLT3 mutation status on patient outcome, the study cohort was divided into the following four groups based on the mutation-testing results at the time of initial diagnosis and at follow-up: 1) a Negative/ Negative group of patients who were negative for FLT3 mutations at the time of initial diagnosis and remained negative at time of all follow-up visits, including during clearly documented hematological relapses; 2) a Positive/Positive group of patients who were positive for FLT3 mutations at the time of initial diagnosis and remained positive at time of all follow-up visits when their bone marrow blast count was at least $5 \%$ (ie, above the sensitivity of our FLT3 assay); 3) a Negative/Positive group of patients who were negative for FLT3 mutation at the time of initial diagnosis but acquired mutation in at least one of their follow-up tests (ie, gained FLT3 mutation during persistent disease after treatment or at relapse); and 4) a Positive/Negative group of patients who were positive for FLT3 mutations at the time of initial diagnosis but became wild type in all followup test, including during documented relapse of acute myeloid leukemia (ie, lost FLT3 mutation). Patients who had received stem cell transplantation or FLT3 inhibitor treatments were excluded. The patients' clinical information, complete blood count, morphological findings in bone marrow, and cytogenetics results were obtained from the medical records. This study was approved by the institutional review board and conducted in accordance with the Declaration of Helsinki.

\section{Cytogenetic and Molecular Analyses}

Conventional cytogenetic analysis was performed on bone marrow aspirate samples in all cases, as described previously. ${ }^{30}$ Karyotypes were reported using the International System for Human Cytogenetic Nomenclature.

Genomic DNA was extracted from fresh bone marrow aspirate samples for FLT3 mutation analysis using the Autopure extractor (QIAGEN/Gentra, Valencia, CA, USA). FLT3-ITD and FLT3-D835 mutations were screened using polymerase chain reaction (PCR) followed by capillary electrophoresis on an Applied Biosystems Prism 3100 Genetic Analyzer (Applied Biosystems, Foster City, CA, USA), as previously described. $^{31}$ For FLT3-D835 point mutation analysis, PCR products were digested with EcoRV before capillary electrophoresis. Sequences were analyzed and mutant allelic ratios were calculated using GeneScan software (Applied Biosystems). The specimens were also analyzed for other genes commonly mutated in acute myeloid leukemia, including NPM1, $K R A S$, and NRAS. These genes were assessed by PCR followed by capillary electrophoresis or direct sequencing (Sanger sequencing or pyrosequencing), according to previously described protocols. ${ }^{31,32}$ The sensitivity of the NPM1 and FLT3 assays is approximately $2.5 \%$. The sensitivity of Sanger sequencing is approximately $20 \%$ and for pyrosequencing, 5-10\%.

\section{Statistical Analysis}

Categorical variables were compared using the Chisquare test. Patient survivals were estimated by the 
a

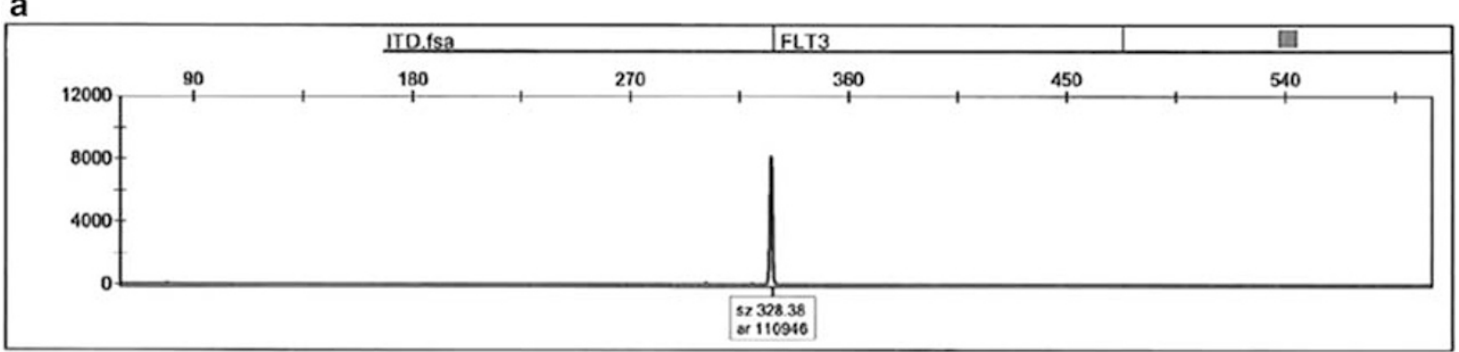

b

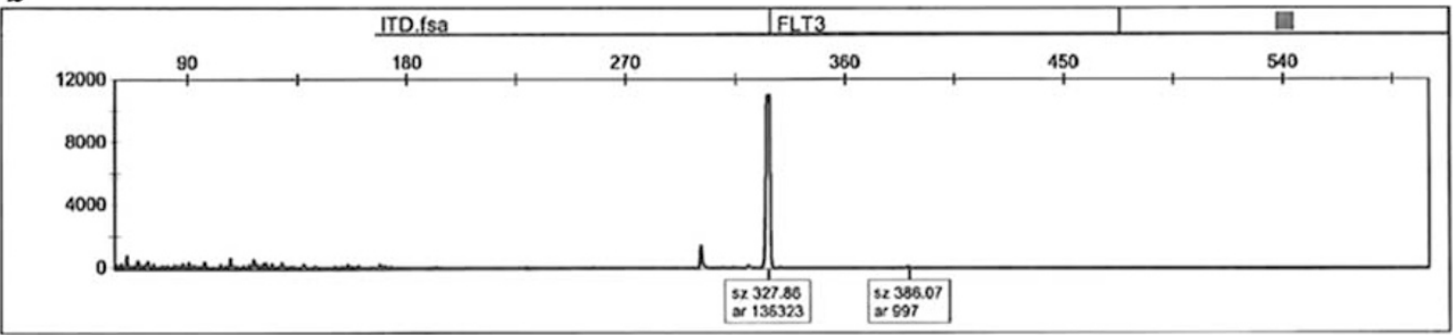

C

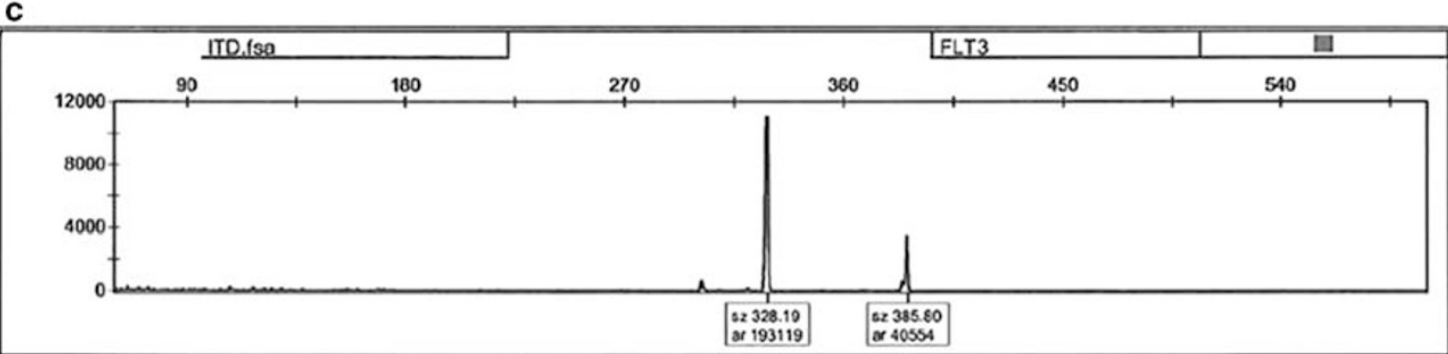

d
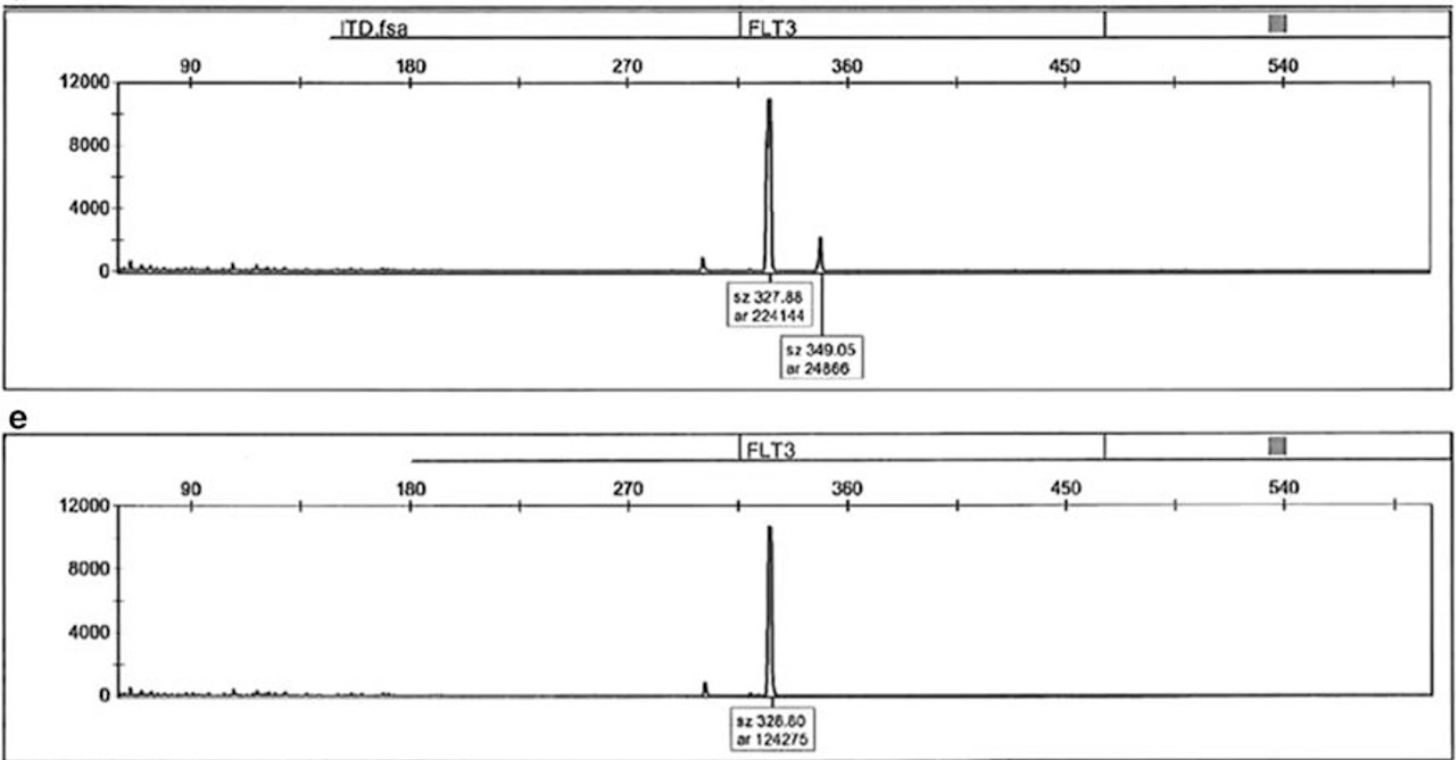

Figure 1 Representative examples of GeneScan results of FLT3 mutation status changes in acute myeloid leukemia patients. A patient diagnosed acute myeloid leukemia with $30 \%$ bone marrow blasts and wild-type FLT3 (a) went into complete remission after treatment, but relapsed 2 months later with 7\% blasts and a detectable FLT3-ITD mutation (b). The patient became resistant to therapy and a bone marrow biopsy 3 months later showed $78 \%$ blasts and more prominent FLT3-ITD mutation (c). Another patient diagnosed acute myeloid leukemia with $46 \%$ bone marrow blasts and FLT3-ITD mutation (d) went into complete remission after treatment. This patient relapsed 5 months later with $23 \%$ blasts and no detectable FLT3 mutation (e). 
Kaplan-Meier method, and differences among groups were determined by the log-rank test.

\section{Results}

\section{FLT3 Mutation Status Changes in a Subset of Acute Myeloid Leukemia Patients}

During our review period of May 2002 to January 2011, a total of 3555 patients were diagnosed with acute myeloid leukemia at our institution and had multiple bone marrow samples tested for FLT3 mutation. Among these patients, 2875 (80.9\%) never tested positive for FLT3 mutation throughout their clinical course, whereas $680(19.1 \%)$ tested positive for FLT3 mutation at least once. These mutated cases included 541 (79.6\%) with FLT3-ITD and 139 $(20.4 \%)$ with FLT3-D835.

In most patients with FLT3 mutation $(n=638$; $93.8 \%$ ), mutation was detected at time of initial diagnosis and the mutation persisted in all followup specimens with $5 \%$ or more blasts. In $42(6.2 \%)$ patients who changed their mutation status, 36 (5.3\%) were negative for FLT3 mutation at initial diagnosis but mutations were later detectable in follow-up samples, including 18 patients with FLT3ITD alone, 11 patients with FLT3-D835 alone, and 7 patients with both types of mutations. These patients were designated as the Negative/Positive group. Another $6(0.9 \%)$ patients were positive for FLT3 mutation at the time of initial diagnosis but subsequently became wild type for FLT3 in all follow-up, including 5 patients with FLT3-ITD alone, and 1 patient with both FLT3-ITD and FLT3-D835. These patients were designated as the Positive/Negative group. Representative results demonstrating the change of mutation status are shown in Figure 1.

\section{FLT3 Status Changes and Clinical Characteristics}

We compared the clinical characteristics and molecular profiles of the 42 patients with a change in FLT3 status to a random selection of 57 patients with persistently negative FLT3 mutation test results (Negative/Negative) as well as 49 randomly selected patients who persistently retained FLT3 mutation in follow-up sample (Positive/Positive). The results are summarized in Table 1.

No statistically significant differences were found with respect to age and gender among these groups. Significantly higher bone marrow blast counts were observed in the FLT3-mutated groups (Positive/ Positive, Negative/Positive, and Positive/Negative), compared with the Negative/Negative group $(P=0.001)$, consistent with earlier studies. ${ }^{6,8}$ The frequency of FLT3-ITD versus FLT3-D835 was similar among the FLT3-mutated groups $(P=0.135)$. The mutant allelic ratio was also similar among these groups $(P=0.676$ and 0.450 for FLT3-ITD and FLT3D835, respectively).

As expected, NPM1 mutations were more frequently seen in FLT3-mutated groups compared with the Negative/Negative group $(P=0.005)$, due to the strong association between these two mutations. RAS mutation rates were similar among all groups (7-14\%) except the Positive/Negative group, in which a higher rate $(33 \%)$ was observed. However, due to the small sample size, the difference was not statistically significant $(P=0.339)$. Additionally, a higher frequency of cytogenetic clonal evolutions was found in patients with FLT3 mutations $(P=0.006)$, especially in patients in the Negative/Positive group.

Patients in the Positive/Positive group had a significant lower complete remission rate than those of the Negative/Negative group $(P=0.003)$. The other two groups of patients with changed

Table 1 Clinopathological characteristics of acute myeloid leukemia patients according to FLT3 mutation statuses at diagnosis and follow-ups

\begin{tabular}{|c|c|c|c|c|c|}
\hline & \multicolumn{4}{|c|}{ FLT3 mutation statuses at diagnosis and follow-ups } & \multirow[t]{2}{*}{ P-value } \\
\hline & $\begin{array}{c}\text { Negative/Negative } \\
\mathrm{n}=57\end{array}$ & $\begin{array}{c}\text { Positive/Positive } \\
\mathrm{n}=49\end{array}$ & $\begin{array}{c}\text { Negative/Positive } \\
\mathrm{n}=36\end{array}$ & $\begin{array}{c}\text { Positive/Negative } \\
\mathrm{n}=6\end{array}$ & \\
\hline Age, median (range), year & $65(22-84)$ & $65(24-91)$ & $64(22-79)$ & $62(18-78)$ & 0.971 \\
\hline Gender, male/female & $32 / 25$ & $24 / 25$ & $17 / 19$ & $4 / 2$ & 0.218 \\
\hline Bone marrow blast count, median (range), \% & $32(20-89)$ & $59(20-94)$ & $51(20-90)$ & $80(48-96)$ & 0.001 \\
\hline \multicolumn{6}{|l|}{ FLT3 mutations: } \\
\hline Mutation type: & & & & & 0.235 \\
\hline ITD only, $n$ & - & 34 & 18 & 5 & \\
\hline D835 only, $n$ & - & 11 & 11 & 0 & \\
\hline ITD and D835, $n$ & - & 4 & 7 & 1 & \\
\hline \multicolumn{6}{|l|}{ Mutant allelic ratio at first detection: } \\
\hline ITD, median (range) & - & $0.44(0.01-27.26)$ & $0.36(0.01-1.14)$ & $0.15(0.01-0.81)$ & 0.676 \\
\hline D835, median (range) & & $0.41(0.06-1.33)$ & $0.19(0.04-1.72)$ & $0.72(0.72-0.72)$ & 0.450 \\
\hline Cytogenetic clonal evolution, $n / N(\%)$ & $8 / 52(15)$ & $15 / 44(34)$ & $16 / 36(44)$ & $0(0)$ & 0.006 \\
\hline NPM1 mutated, $n(\%)$ & $1(2)$ & $9(18)$ & $4(11)$ & $0(0)$ & 0.005 \\
\hline RAS mutated, $n(\%)$ & $4(7)$ & $7(14)$ & $5(13)$ & $2(33)$ & 0.339 \\
\hline Complete remission rate, \% & 81 & 68 & 94 & 100 & 0.003 \\
\hline
\end{tabular}


FLT3 mutation status showed complete remission rates closer to that of the Negative/Negative group. However, the higher complete remission rates in these two groups may be biased due to the fact that our patient selection criteria excluded patients without follow-up testing, and most of the patients who did not reach a complete remission died before they were re-tested for FLT3. As a result, the majority of these two groups were patients who went into complete remission and then relapsed.

\section{FLT3 Status Changes Impact Patient Survival}

The Kaplan-Meier curves plotting the overall survival among the four study groups are shown in Figure 2a. Patients who gained mutations (Negative/ Positive) had an overall survival similar to that of the Negative/Negative group $(P=0.464)$, and was significant better than that of the Positive/Positive group $(P<0.001)$. The best survival appeared to be the six patients in the Positive/Negative group who lost FLT3 mutation after first complete remission, although admittedly the sample size is too small to accurately predict their behavior. However, when examining survival time of the Negative/Positive patients after they turned positive for FLT3 mutations, their survival curve tracked that of the Positive/Positive group after their first relapse (Figure 2b; $P=0.761$ ), and was significantly worse than that of the Negative/Negative group $(P<0.001)$. In the Negative/Positive group, the median lag time from initial diagnosis without FLT3 mutation to testing positive for mutation was 15 months, ranging from 2 to 45 months. The survival, therefore, became dramatically worse for these patients after gaining FLT3 mutation and all but one patient died shortly after FLT3 mutation was acquired.

We further compared the survival of patients with FLT3-ITD and those with FLT3-D835 mutation in the Positive/Positive and Negative/Positive groups (Figure 3). No significant difference was found between acute myeloid leukemia patients with these two types of mutations in either overall survival or survival time after first relapse in Positive/Positive group or after FLT3 mutation becoming positive in Negative/Positive group.

\section{Discussion}

It is well established that FLT3 mutation is an important prognostic factor in patients with acute myeloid leukemia. Therefore, accurate assessment of FLT3 mutation status is crucial to risk stratification, clinical management, and treatment selection for acute myeloid leukemia patients. Several studies have observed FLT3 mutation status changes in small subsets of acute myeloid leukemia patients and have implied the prognostic importance. ${ }^{10,16,21-29}$ In aggregate, these data suggest that gain of FLT3
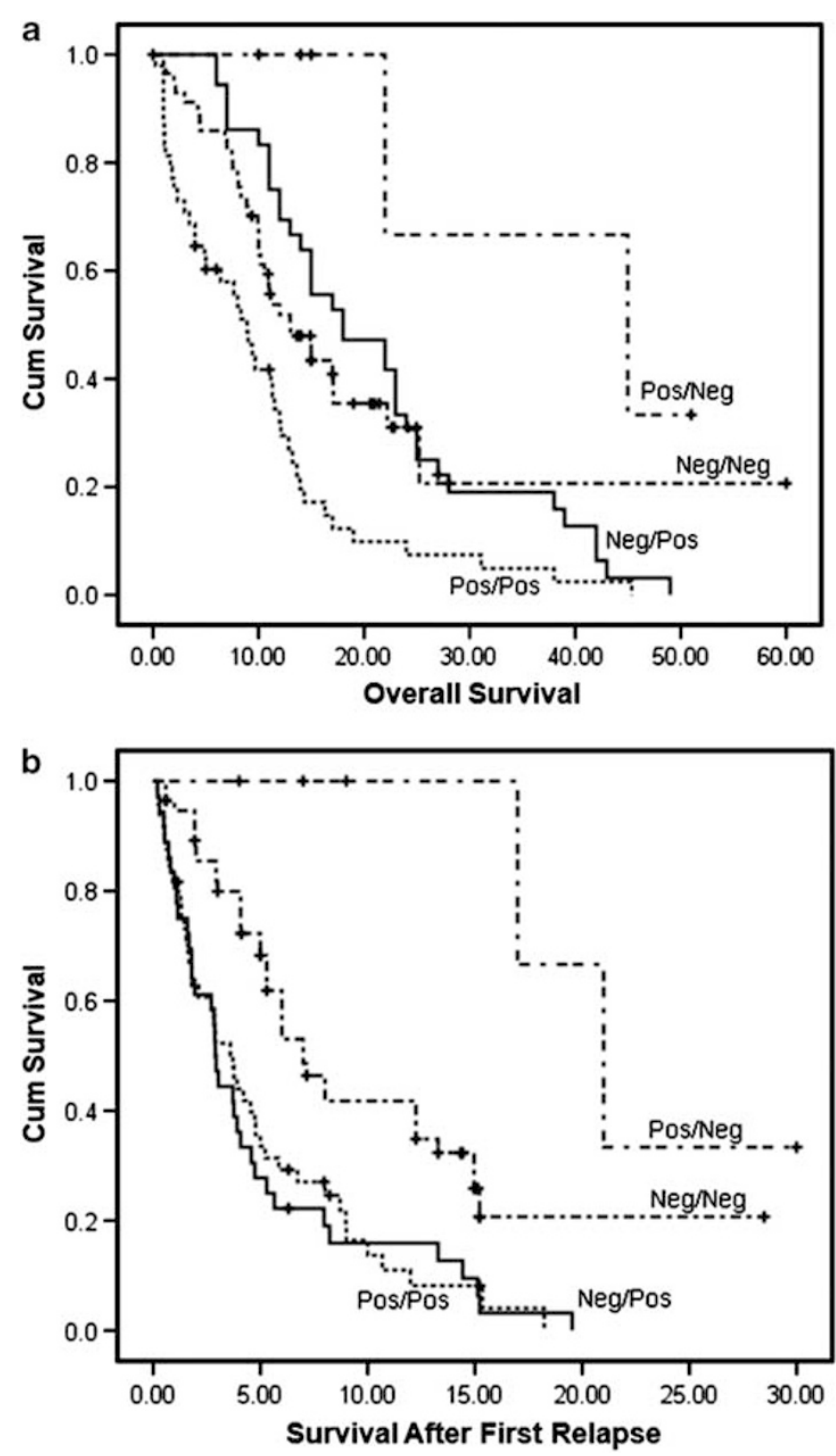

Figure 2 Survival of acute myeloid leukemia patients according to FLT3 mutation statuses at diagnosis and follow-ups. (a) Overall survival of all patients in the four groups $(P<0.001)$. (b). Survival after first relapse or after FLT3 mutation detected in the Negative/ Positive group $(P<0.001)$.

mutations may be associated with worse prognosis. ${ }^{24,27,29,33}$ These patients also seem not to benefit from intensifying chemotherapy. ${ }^{34-36}$ The number of patients in these studies, however, are truly small. We present the first study focused on a large group of such patients, thereby making it possible to statistically analyze clinical characteristics and the prognostic impact of a change in FLT3 status.

In this study, approximately $6 \%$ of acute myeloid leukemia patients had a demonstrated change in FLT3 status during their disease course, similar to reports in earlier studies. ${ }^{10,16,21-29}$ One previous study reported that FLT3-ITD is more often acquired than FLT3-TKD at time of relapse, $8 \%$ and $2 \%$, respectively, whereas FLT3-TKD is more often lost 

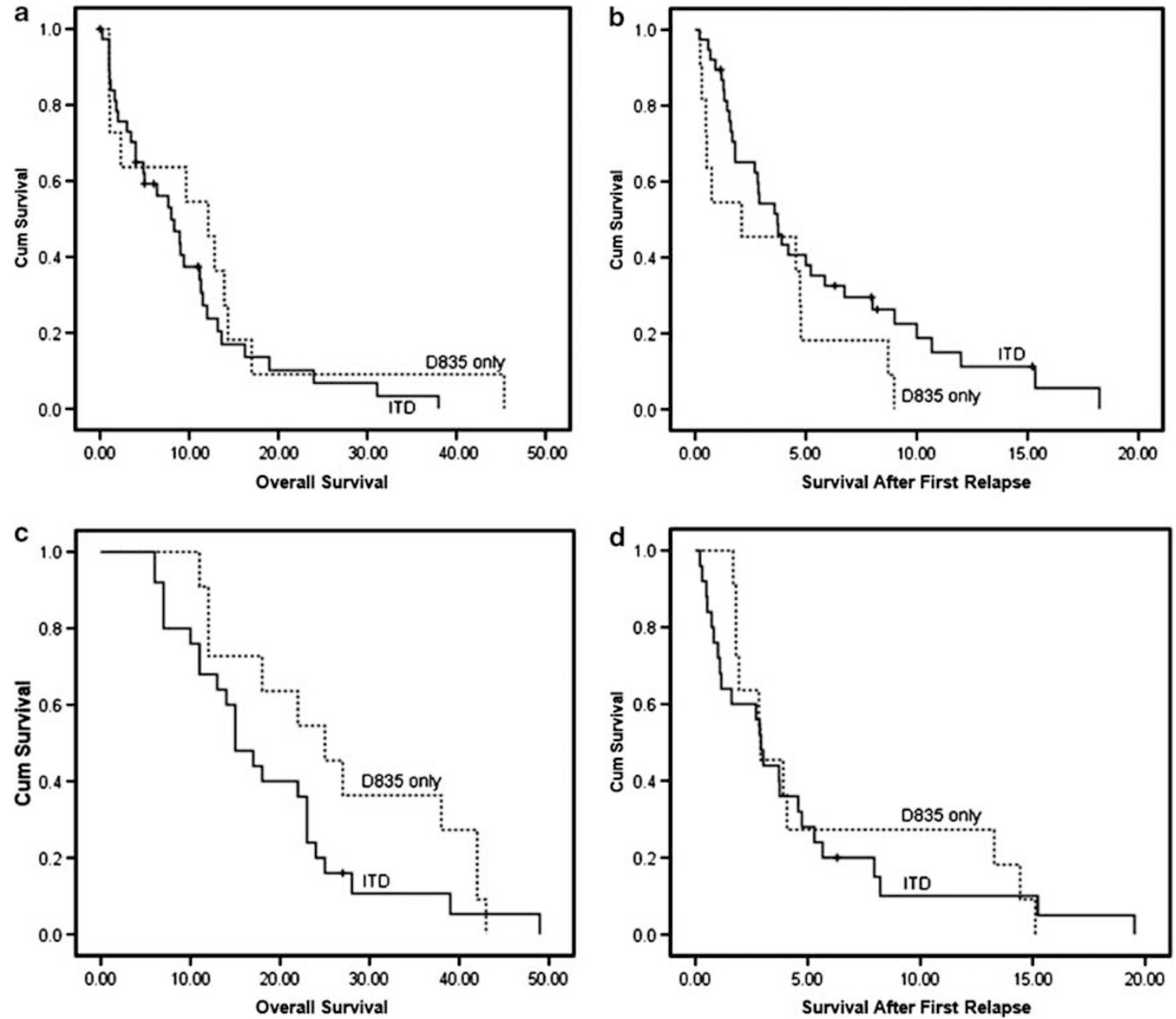

Figure 3 Comparaison of survival between acute myeloid leukemia patients with FLT3-ITD mutation and those with FLT3-D835 mutation. (a) Overall survival in the Positive/Positive groups $(P=0.169)$. (b) Survival after first relapse in the Positive/Positive groups $(P=0.780)$. (c) Overall survival in the Negative/Positive groups $(P=0.086)$. (d) Survival after FLT3 mutations detected in the Negative/ Positive groups $(P=0.368)$.

than FLT3-ITD, $7 \%$ and $4 \%$, respectively. ${ }^{29}$ In our study, FLT3 mutations were acquired far more often than lost, in a ratio of 6 to 1, during the disease course. In addition, FLT3-ITD was more often involved than FLT3-TKD in both patients with gain or loss of FLT3 mutation.

We compared the clinical presentation of patients with a change in FLT3 mutation status to randomly chosen groups of patients without change in FLT3 status. There was no significant difference in age and gender distribution between these groups. However, patients who gained the mutations during follow-up (Negative/Positive group) presented with a higher average bone marrow blast count. The Negative/Positive group also had a higher frequency of NPM1 mutations compared with the Negative/ Negative group. Although the predictive power of these two findings is low, given the high frequency of a high blast count and NPM1 mutation, and the relative low frequency of a change in FLT3 status, these features in a patient presented with wild-type
FLT3 may serve as an early indicator for possible emergence of FLT3 mutations.

Importantly, our results show that a gain of FLT3ITD mutation strongly correlates with disease progressions and worse prognosis. Patients in the Negative/Positive group initially enjoyed similar survival rate as patients in the Negative/Negative group, even after relapse, but this was no longer the case once a FLT3 mutation became detectable. Affected patients showed dramatic exacerbations of their disease after relapse with FLT3 mutation and commonly died shortly thereafter. The median survival of these patients after acquiring a FLT3 mutation was very similar to that of patients in the Positive/Positive group after first relapse, often less than 5 months post first relapse. ${ }^{34,36}$ Based on these results, we suggest that all acute myeloid leukemia patients be tested repeatedly for FLT3 mutation over their disease course to monitor the mutation status.

The exact mechanism of the change in FLT3 mutation status is not clear. One possibility is that 
FLT3 mutations may emerge owing to the instability of the tumor genome. ${ }^{24,25}$ The higher frequency of cytogenetic clonal evolution known to be associated with FLT3 mutations found in this study potentially supports this possibility. A second possibility is that, leukemia cells with FLT3 mutations may be present in bone marrow at time of initial diagnosis in a very small number that is below the level of detection of the assays to assess FLT3. These FLT3mutated leukemia cells have a survival advantage over their wild-type counterparts and eventually become a dominant clone. There is evidence that conventional therapies for acute myeloid leukemia appear to give a selection advantage to leukemia cells depending on FLT3 signaling, and resulting in increased expression of the mutant allele or loss of the wild-type allele at relapse. ${ }^{37}$ This phenomenon may be related to clonal expansion after therapy. Findings in this study also show that emergence of FLT3-mutated leukemia cells in the Negative/Positive patient group correlated with a poor response to conventional chemotherapy, significantly impacting patient survival in a negative manner. Therefore, change in FLT3 mutation status has practical implications for patient management, and is not simply an academic issue.

Conventional chemotherapy is not effective in eradicating FLT3-mutated leukemia cells. In our study, only 6 of 644 patients who were initially positive for FLT3 mutations successfully eliminated these mutations during their disease course, presumably as a result of conventional therapy alone. Other studies also suggest that FLT3 signaling may be a key survival mechanism in leukemia cells. ${ }^{38}$ Overexpression of FLT3 also has been detected in acute myeloid leukemia patients without FLT3 mutations and it is associated with a poor overall survival. ${ }^{39}$ These data argue that patients with FLT3 mutations may require their own therapeutic regimens, including FLT3 inhibitors, agents that target the aberrantly activated FLT3 kinase. In fact, FLT3 inhibitors have attracted broad attention as new therapeutic agents for acute myeloid leukemia, and there are multiple clinical trials that are currently in progress. Both small molecules and FLT3-directed antibodies are currently in trials. ${ }^{19}$ It has been suggested that all acute myeloid leukemia patients might benefit from receiving FLT3 inhibitor regimens starting from their initial diagnosis regardless of their mutation status. ${ }^{20,37}$ Patients in the Negative/ Positive group in our study would support this view.

In summary, this study demonstrates that a change of FLT3 status occurs in a small subset of acute myeloid leukemia patients, approximately $6 \%$. As a result of the large cohort of AML patients at our institution, we have reported on 42 patients who showed a change in FLT3 status. Importantly, acquisition of FLT3 mutation has a negative clinical impact on survival. The findings suggest that FLT3 mutations are unstable and that acute myeloid leukemia cells are constantly evolving. Our data suggest that continuous testing for FLT3 mutation throughout the disease course is important, and may be most important for patients that initially tested negative for FLT3 mutation. Adding FLT3 inhibitors to standard therapeutic regimens may be beneficial for all patients who suffer from acute myeloid leukemia.

\section{Acknowledgement}

The authors appreciate the excellent support of Gloria Rice in preparing the manuscript.

\section{Disclosure/conflict of Interst}

The authors declare no conflict of interest.

\section{References}

1 Drexler HG. Expression of FLT3 receptor and response to FLT3 ligand by leukemic cells. Leukemia 1996;10:588-599.

2 Gilliland DG, Griffin JD. The roles of FLT3 in hematopoiesis and leukemia. Blood 2002;100: 1532-1542.

3 Stirewalt DL, Radich JP. The role of FLT3 in haematopoietic malignancies. Nat Rev Cancer 2003;3:650-665.

4 Nakao M, Yokota S, Iwai $\mathrm{T}$, et al. Internal tandem duplication of the flt3 gene found in acute myeloid leukemia. Leukemia 1996;10:1911-1918.

5 Kiyoi H, Towatari M, Yokota S, et al. Internal tandem duplication of the FLT3 gene is a novel modality of elongation mutation which causes constitutive activation of the product. Leukemia 1998;12:1333-1337.

6 Kottaridis PD, Gale RE, Frew ME, et al. The presence of a FLT3 internal tandem duplication in patients with acute myeloid leukemia (AML) adds important prognostic information to cytogenetic risk group and response to the first cycle of chemotherapy: analysis of 854 patients from the United Kingdom Medical Research Council AML 10 and 12 trials. Blood 2001;98:1752-1759.

7 Yamamoto Y, Kiyoi H, Nakano Y, et al. Activating mutation of D835 within the activation loop of FLT3 in human hematologic malignancies. Blood 2001; 97:2434-2439.

8 Thiede C, Steudel C, Mohr B, et al. Analysis of FLT3activating mutations in 979 patients with acute myelogenous leukemia: association with FAB subtypes and identification of subgroups with poor prognosis. Blood 2002;99:4326-4335.

9 Kiyoi H, Naoe T, Yokota S, et al. Internal tandem duplication of FLT3 associated with leukocytosis in acute promyelocytic leukemia. Leukemia Study Group of the Ministry of Health and Welfare (Kohseisho). Leukemia 1997;11:1447-1452.

10 Schnittger S, Schoch C, Dugas M, et al. Analysis of FLT3 length mutations in 1003 patients with acute myeloid leukemia: correlation to cytogenetics, FAB subtype, and prognosis in the AMLCG study and usefulness as a marker for the detection of minimal residual disease. Blood 2002;100:59-66. 
11 Bacher U, Haferlach C, Kern W, et al. Prognostic relevance of FLT3-TKD mutations in AML: the combination matters-an analysis of 3082 patients. Blood 2008;111:2527-2537.

12 Chen W, Jones D, Medeiros LJ, et al. Acute myeloid leukaemia with FLT3 gene mutations of both internal tandem duplication and point mutation type. Br J Haematol 2005;130:726-728.

13 Brandts CH, Sargin B, Rode M, et al. Constitutive activation of Akt by Flt3 internal tandem duplications is necessary for increased survival, proliferation, and myeloid transformation. Cancer Res 2005;65: 9643-9650.

14 Santos FP, Jones D, Qiao W, et al. Prognostic value of FLT3 mutations among different cytogenetic subgroups in acute myeloid leukemia. Cancer 2011;117: 2145-2155.

15 Abu-Duhier FM, Goodeve AC, Wilson GA, et al. FLT3 internal tandem duplication mutations in adult acute myeloid leukaemia define a high-risk group. Br J Haematol 2000;111:190-195.

16 Kiyoi H, Naoe T, Nakano Y, et al. Prognostic implication of FLT3 and N-RAS gene mutations in acute myeloid leukemia. Blood 1999;93:3074-3080.

17 Abu-Duhier FM, Goodeve AC, Wilson GA, et al. Identification of novel FLT-3 Asp835 mutations in adult acute myeloid leukaemia. $\mathrm{Br} \mathrm{J}$ Haematol 2001;113:983-988.

18 Whitman SP, Ruppert AS, Radmacher MD, et al. FLT3 D835/I836 mutations are associated with poor diseasefree survival and a distinct gene-expression signature among younger adults with de novo cytogenetically normal acute myeloid leukemia lacking FLT3 internal tandem duplications. Blood 2008;111:1552-1559.

19 Levis MJ. Will newer tyrosine kinase inhibitors have an impact in AML? Best Pract Res Clin Haematol 2011;23:489-494.

20 Knapper S, Burnett AK, Littlewood T, et al. A phase 2 trial of the FLT3 inhibitor lestaurtinib (CEP701) as first-line treatment for older patients with acute myeloid leukemia not considered fit for intensive chemotherapy. Blood 2006;108:3262-3270.

21 Suzuki T, Kiyoi H, Ozeki K, et al. Clinical characteristics and prognostic implications of NPM1 mutations in acute myeloid leukemia. Blood 2005;106: 2854-2861.

22 Schnittger S, Schoch C, Kern W, et al. FLT3 length mutations as marker for follow-up studies in acute myeloid leukaemia. Acta Haematol 2004;112:68-78.

23 Cloos J, Goemans BF, Hess CJ, et al. Stability and prognostic influence of FLT3 mutations in paired initial and relapsed AML samples. Leukemia 2006;20:1217-1220.

24 Kottaridis PD, Gale RE, Langabeer SE, et al. Studies of FLT3 mutations in paired presentation and relapse samples from patients with acute myeloid leukemia: implications for the role of FLT3 mutations in leukemogenesis, minimal residual disease detection, and possible therapy with FLT3 inhibitors. Blood 2002;100:2393-2398.
25 Shih LY, Huang $\mathrm{CF}, \mathrm{Wu} \mathrm{JH}$, et al. Internal tandem duplication of FLT3 in relapsed acute myeloid leukemia: a comparative analysis of bone marrow samples from 108 adult patients at diagnosis and relapse. Blood 2002;100:2387-2392.

26 Shih LY, Huang CF, Wu JH, et al. Heterogeneous patterns of FLT3 Asp(835) mutations in relapsed de novo acute myeloid leukemia: a comparative analysis of 120 paired diagnostic and relapse bone marrow samples. Clin Cancer Res 2004;10:1326-1332.

27 Tiesmeier J, Muller-Tidow C, Westermann A, et al. Evolution of FLT3-ITD and D835 activating point mutations in relapsing acute myeloid leukemia and response to salvage therapy. Leuk Res 2004;28: 1069-1074.

28 Palmisano M, Grafone T, Ottaviani E, et al. NPM1 mutations are more stable than FLT3 mutations during the course of disease in patients with acute myeloid leukemia. Haematologica 2007;92:1268-1269.

29 McCormick SR, McCormick MJ, Grutkoski PS, et al. FLT3 mutations at diagnosis and relapse in acute myeloid leukemia: cytogenetic and pathologic correlations, including cuplike blast morphology. Arch Pathol Lab Med 2010;134:1143-1151.

30 Alseraye FM, Zuo Z, Bueso-Ramos C, et al. Trisomy 11 as an isolated abnormality in acute myeloid leukemia is associated with unfavorable prognosis but not with an NPM1 or KIT mutation. Int J Clin Exp Pathol 2011;4:371-377.

31 Bains A, Luthra R, Medeiros LJ, et al. FLT3 and NPM1 mutations in myelodysplastic syndromes: frequency and potential value for predicting progression to acute myeloid leukemia. Am J Clin Pathol 2011;135:62-69.

32 Zuo Z, Chen SS, Chandra PK, et al. Application of COLD-PCR for improved detection of KRAS mutations in clinical samples. Mod Pathol 2009;22:1023-1031.

33 Griffiths M, Mason J, Rindl M, et al. Acquired isodisomy for chromosome 13 is common in AML, and associated with FLT3-itd mutations. Leukemia 2005;19:2355-2358.

34 Ravandi F, Kantarjian H, Faderl S, et al. Outcome of patients with FLT3-mutated acute myeloid leukemia in first relapse. Leuk Res 2010;34:752-756.

35 Griffiths EA, Gore SD, Hooker CM, et al. Epigenetic differences in cytogenetically normal versus abnormal acute myeloid leukemia. Epigenetics 2010;5:590-600.

36 Levis M, Ravandi F, Wang ES, et al. Results from a randomized trial of salvage chemotherapy followed by lestaurtinib for patients with FLT3 mutant AML in first relapse. Blood 2011;117:3294-3301.

37 Levis M. FLT3/ITD AML and the law of unintended consequences. Blood 2011;117:6987-6990.

38 Knapper S, Mills KI, Gilkes AF, et al. The effects of lestaurtinib (CEP701) and PKC412 on primary AML blasts: the induction of cytotoxicity varies with dependence on FLT3 signaling in both FLT3-mutated and wild-type cases. Blood 2006;108:3494-3503.

39 Ozeki K, Kiyoi H, Hirose Y, et al. Biologic and clinical significance of the FLT3 transcript level in acute myeloid leukemia. Blood 2004;103:1901-1908. 